\title{
Respiratory Viral Infections in Cancer Patients: Epidemiological, Clinical and Cost-Effective Study from a Developing Country
}

Nitin Bansal ( $\sim$ nitin.bansal3011@gmail.com )

Rajiv Gandhi Cancer Institute

Neelam Sachdeva

Rajiv Gandhi Cancer Institute

Dinesh Bhurani

Rajiv Gandhi Cancer Institute

Gauri Kapoor

Rajiv Gandhi Cancer Institute

Pinky Yadav

Rajiv Gandhi Cancer Institute

Sumit Goyal

Rajiv Gandhi Cancer Institute

Vineet Talwar

Rajiv Gandhi Cancer Institute

Ullas Batra

Rajiv Gandhi Cancer Institute

\section{Research Article}

Keywords: Respiratory Viral Infections, Oncology, Febrile Neutropenia, Antibiotic Stewardship, Antibiotic De-escalation

Posted Date: January 19th, 2022

DOI: https://doi.org/10.21203/rs.3.rs-1266819/v1

License: (c) (i) This work is licensed under a Creative Commons Attribution 4.0 International License. Read Full License 


\section{Abstract}

Background: Diagnosis of respiratory viral pathogens can lead to therapeutic changes, improvement in infection control practices and reduction in use of antibiotics.

Methods: This is a retrospective study which looked into the case records of patients (June 2020-Dec 2021) who were diagnosed with a respiratory viral infection. Demographic, clinical and antibiotic prescription patterns of these patients were studied. Inter-group analysis was done between antibiotic deescalation cohort and non-descalation cohort.

Results: Total of 57 patients were identified with median age of 40 years (range: 3-68), out of which 38 $(66.6 \%)$ were males. Hematological malignancies were present in $45(78.9 \%)$ of patients, whereas 14 (24.5\%) patients were stem cell transplant recipients. Severe neutropenia was seen in $32(56.1 \%)$ patients with median duration of neutropenia of 7 (range 5-20) days. RSV (36.8\%) was the most common virus detected followed by rhinovirus (19.3\%) and influenza (19.3\%). Antibiotic de-escalation was done in 33 (57.8\%) of patients and in $13(22.8 \%)$ bacterial co-infection was diagnosed. More than $3 / 4^{\text {th }}$ patients were shifted to isolation and nearly 9000 INR per patient were saved by de-escalation of antibiotics. Death occurred in 4 (7.0\%) patients. On inter-group analysis, non-de-escalation cohort had longer length of stay ( 10 vs 3 days; $p=0.005$ ), no antibiotic cost reduction ( 0 vs $9620 ; p=0.0003$ ), higher mortality $(16.6 \%$ vs $0 \% ; p=0.01)$ \& higher rhinovirus detection rates $(41.6 \%$ vs $3 \% ; p=0.0002)$.

Conclusion: Diagnosis of respiratory viral infection helped in improving antibiotic stewardship and infection control practices.

\section{Introduction}

Management of infection in patients who are undergoing cancer therapy is challenging from clinicians, hospital administrators and patient point of view. Cancer therapy and sometimes malignancy per-se can make these patients vulnerable to multiple infections. Since cancer therapy is prolonged and treatment duration may range from months to years, these patients may get infected multiple times, which makes these patients physically, emotionally and financially vulnerable.

Infection by respiratory viruses is very common in oncological settings and throws unique challenges in diagnostic, clinical management, and infection control aspects. Diagnosis of respiratory viral pathogen relies heavily on molecular technology, which is not widely available across Indian clinical laboratories. But, with COVID-19 pandemic, importance of quick and reliable diagnosis of a respiratory viral pathogen has been emphasized. Our centre (tertiary care dedicated cancer centre in north India), strengthened its diagnostics by acquiring 2 multiplex panel (Biofire- detects 23 pathogens \& Cephied Xpert Xpress FLU/RSV- detects 3 pathogens), both of which have found to have good sensitivity, specificity and good clinical impact. ${ }^{1,2}$ 
Diagnosing, a respiratory viral infection in oncology patients opens up another few sets of dilemmas, particularly in a patient who is having severe neutropenia. Antibiotic de-escalation after a diagnosis of respiratory viral pathogen in febrile neutropenic patient has not been well studied and various guidelines either recommend clinicians' discretion or avoid giving any recommendation. ${ }^{3}$ In the era of multi-drug resistant gram-negatives, it is also imperative to practice good antibiotic stewardship (AMS) practices and many published articles exist which prove that AMS leads to good clinical outcomes in oncological

settings. ${ }^{4,5}$ Further, these patients need to be shifted to transmission based (contact, droplet or airborne) isolation precautions, which require single rooms with dedicated infrastructure and man power. ${ }^{6}$ Many Indian hospitals including ours have mostly multi-bedded wards with few single rooms.

With this in background, we planned this study with objectives of understanding the epidemiology and clinical outcomes of patients who were detected to have a respiratory viral infection and also change in the antibiotic prescription practices and cost savings (if any) in these patients.

\section{Methodology}

It was a retrospective, single center study conducted in a tertiary care centre in North India. We reviewed case records of patients whose respiratory tract sample was found to have a respiratory virus from the period of June 2020 till Dec 2021. We collected demographic data (age and sex), clinical features (baseline malignancies, clinical presentation, neutropenia), radiological features, intervention done by the clinician (antibiotic prescription changes, adding of specific therapy for viral infection), antibiotic cost savings, clinical outcomes and change in infection control practices after a respiratory viral infection was diagnosed. We excluded patients with SARS COV-2 as standard treatment has changed over time as pandemic has evolved and requires a separate analysis all together.

We divided our cohort into two groups. Group A comprised on patients in whom antibiotics were deescalated upon detection of respiratory viral infection and Group B comprised of patients in whom antibiotics were not de-escalated or escalated. We did an intergroup analysis of these two groups and used student $t$ test and chi-square test to study various parameters.

\section{$\underline{\text { Antibiotic cost calculation }}$}

Antibiotics prescribed to the patients are of different brands with varying costs so for uniformity in calculation, brand with lowest cost were taken (Details in supplementary Table). It was assumed that a patient would have got the prescribed antibiotic for at least 7 days if the viral pathogen was not detected (a common practice in oncology settings particularly in neutropenia). Cost of antibiotic saved was calculated (for a particular patient) as per following formula:

Cost saved (for a particular patient) $=$ Total cost of antibiotic prescribed for 7 days - Cost of antibiotic which that patient actually received 
De-escalation of antibiotic was defined as one of the following; a) stopping all antibiotics b) shifting from a broad spectrum agent to a narrow spectrum agent.

\section{Results}

We found a total of 57 patients (Table 1) which were found a respiratory viral panel detected in their respiratory sample (nasopharyngeal or oropharnygeal swab or a bronchoscopic alveolar lavage) during the study period. Median age of patients was 40 years (range: $3-68)$, out of which 38 (66.6\%) were males. Hematological malignancies $(45 ; 78.9 \%)$ were most common followed by solid organ tumors $(10 ; 17.5 \%)$. Non-oncological conditions like thalessemia and aplastic anemia was seen in 2 patients. Total of 14 (24.5\%) patients got respiratory viral infection after stem cell transplant, out of which 4 got the diagnosis of the respiratory viral infection before engraftement. Median days post transplant was 37 days (range: 5400).

All patients had one or more respiratory complaints (cough, sore-throat and/or breathlessness). Close to $80 \%$ of patients also had fever. Severe neutropenia $\left(\right.$ ANC $\left.<500 \mathrm{~mm}^{3}\right)$ was present in $32(56.1 \%)$ at the time of diagnosis of the respiratory viral infection and 30 (52.6\%) patients fulfilled the criteria of febrile neutropenia. Median duration of neutropenia was 7 days (range: 5-20). ICU management was required in $6(10.5 \%)$ patients and $15(26.3 \%)$ patients required supplemental oxygen support.

Most cases were seen in month of July 2021-Oct 2021; 39 (68.4\%) cases in period of 4 months (Figure 1). Positive yield of the test ranged from $0-66.6 \%$, but it was highest in month of October 2021 (66.6\%).

All patients got chest imaging (X-ray and/or CT scan) done during their course of stay in the hospital. Chest imaging was normal in $27(47.3 \%)$ of patients, where as ground glass opacities were seen in 22 (38.5\%), nodules in 2 (3.5\%) and lobar consolidation in 4 (7.0\%) patients.

Respiratory syncytial virus was most commonly detected virus, seen in $21(36.8 \%)$ patients, followed by Influenza $(11 ; 19.3 \%)$ and rhinovirus $(11 ; 19.3 \%)$ patients. No specific anti-viral drug was added in 36 patients, where as all patients diagnosed with influenza were given oral oseltamivir. Only $6(28.5 \%)$ patients diagnosed with RSV were given oral ribavirin. Polyclonal intravenous immunoglobulin (IVIG) was given to 4 patients; whereas one patient received systemic steroids after respiratory virus related acute respiratory distress syndrome (ARDS) was diagnosed.

All patients were on antibiotics at the time of diagnosis of respiratory viral infection. Antibiotic deescalation was done in $33(57.8 \%)$ of patients, whereas 14 (24.5\%) patients did not get any change in antibiotic prescription despite the diagnosis of a respiratory infection. Ten (17.5\%) patients had their antibiotics escalated as they were diagnosed to have bacterial co-infection which was resistant to antibiotic they were on. Median duration of antibiotic given was 2 days (range: 1-14), only 7 (12.2\%) patients got antibiotics for more than 7 days. 
Transmission based precautions (contact for RSV and droplet for influenza, human metapneumovirus and para-influenza virus) were applied for $43(75.4 \%)$ patients. None of the patients with rhinovirus were shifted to transmission based precautions. Three patients with RSV were not shifted to isolation due to practical reasons (lack of single room availability, discharged on same day etc,)

As per our antibiotic cost calculation, we found that a median of 9000 INR (range 0-67500) were saved per patient after they were diagnosed with a respiratory viral infection which led to change in antibiotic prescription. Median duration of stay in the hospital was 6 days (range: 1-22). Four (7.0\%) patients died in the study cohort.

We also did an intergroup analysis (Table 2) in two groups (antibiotic de-escalation cohort and non-deescalation cohort). We found that non-de-escalation cohort had longer length of stay (10 vs 3 days; $p$ $=0.005$ ), no antibiotic cost reduction ( 0 vs $9620 ; p=0.0003)$, higher mortality $(16.6 \%$ vs $0 \% ; p=0.01)$ \& higher rhinovirus detection rates $(41.6 \%$ vs $3 \% ; p=0.0002)$, but that was partially explained by higher rates of bacterial co-infection in that cohort ( $54 \%$ vs $0 \% ; p=0.0002)$.

\section{Discussion}

Utilizing a multiplex PCR panel for respiratory pathogen may be a standard of care in a developed world, but it is not commonly utilized in low-middle income countries primarily because of high cost. Lack of good therapeutic interventions, evidence to de-escalate antibiotics and isolation infra-structure are some other reasons which make it cost-ineffective. COVID-19 pandemic has taught us many lessons, some of which include the importance of confirmation of clinical diagnosis and emphasis on infection control practices particularly in a dedicated oncology centre which deals with immunocompromised patients and cross-transmission can lead to devastating results. Due to these lessons, our institute strengthened its viral diagnostics, improved our antibiotic prescriptions patterns and came-up with more effective infection control practices.

There are multiple studies which have looked into the impact of diagnosing respiratory viral infections in oncology settings and have found varied results. ${ }^{7-9}$ Even IDSA guidelines on the use of same, fail to give a strong recommendation and only conclude that utilization of these molecular tests should be done in consideration with regards to hospital epidemiology and antibiotic stewardship. They also highlight lack of good randomized control trials which can answer these unmet needs. ${ }^{10}$

Our study found that in $>50 \%$ of patients, there was no specific antiviral added, that may be because of most patients were infected with RSV and Rhinovirus. Data of use of oral ribavirin for RSV is very heterogeneous and guidelines suggest its use in patients with high risk of progression to lower respiratory tract. ${ }^{11,12}$ Apart from influenza, and probably RSV, most other respiratory viruses do not have an approved antiviral agent as of now. This brings us to a point of therapeutic efficacy of doing the test (therapy change after testing), we do agree, addition of specific antiviral agent may not be possible in most the patients, but in a patient with respiratory symptoms and signs compatible with a respiratory viral 
infection and a positive respiratory viral result, it becomes easy to de-escalate antibiotics. In the era of multi-drug resistance gram negatives, management of febrile neutropenia has become even more challenging. Judicious use of antibiotics have shown good outcomes in oncology patients and most guidelines now recommend intervention by AMS team in management of febrile neutropenia. ${ }^{4,5}$ Multidrug resistant gram negative is a major problem in India and oncology patients are most adversely affected by it. It is imperative that AMS is encouraged in India and this study makes an attempt towards that. Neutropenia was seen in more than half of our study population and still clinicians de-escalated antibiotics in close to $57 \%$ patients.

Change in infection control practices (shifting to transmission based precautions) is only possible after a diagnosis of respiratory infection has been made. Following good infection control practices is of utmost importance particularly in oncology settings as cross-transmission can lead to outbreaks with poor patient related outcomes. We were able to shift more than $75 \%$ of our patients to isolation rooms, providing a good example for hospital administrators to invest in good infection control infrastructure.

So, we feel if good antibiotic stewardship and infection control practices are put in place, then therapeutic efficacy of doing respiratory viral panels is very good particularly in immunocompromised hosts. Cost of antibiotic (median saving of 9000 INR per patient in our study) saved adds to the cost effectiveness of these tests. In a study done by Shinn et. al, they concluded that there was no difference in patient related outcomes in between patients with positive and negative viral panel results. ${ }^{9}$ High incidence of detecting rhinovirus (61\%) may have been the reason for this outcome. In our study also, patients in whom antibiotics were not de-escalated (most commonly due to presence of bacterial co-infection) had high rates of rhinovirus detection $(41.6 \% \mathrm{vs} 3 \% ; \mathrm{p}=0.0002)$ and other patients related outcomes like length of stay, mortality and cost of antibiotics saved were badly affected (Table 2 ). Although human rhinovirus can cause severe pneumonia but it has also been reported as a nasal commensal ${ }^{13}$, so we recommend caution while interpreting reports with positive rhinovirus results.

Most of our cases were diagnosed in the July 2021-Octover 2021, this period of 4 months lead to over $68 \%$ of cases and also positive yield of doing the tests was also highest during this period (42.8\%-66.6\%). This may be because of heavy rains (monsoon) during this time of year, while this is in line with the national data, same was not observed in 2020 as pandemic led to a lockdown which may have affected the spread of respiratory viral infections.

Our study has many limitations. Firstly, it lacks a true comparator arm of patients in whom respiratory panel was negative, which could have given our study findings more credibility. Secondly, cost calculation of antibiotics is based on an assumption (7 days if virus test was not done). Lastly, it being a retrospective study, suffers from selection bias. Despite the limitations, our study gives a 'real life' insight into clinicians' behavior after he/she finds a patient with proven respiratory viral infection in oncology settings. It also encourages antibiotic stewardship behavior among oncologists and promotes developing a culture for good infection control practices. 


\section{Conclusion}

Diagnosis of a respiratory viral pathogen helps in promoting antibiotic stewardship and infection control practices. However, caution needs to be taken while interpreting results with rhinovirus.

\section{Abbreviations}

RSV - Respiratory Syncytial Virus

INR - Indian National Rupee

AMS - Anti-microbial stewardship

ANC - Absolute Neutrophill count

PCR- Polymerase Chain Reaction

IDSA - Infectious Diseases Society of America

\section{Declarations}

\section{Ethics and consent}

Study was approved by Institute Ethics Committee (IEC) and patient consent was waived off as it is a retrospective study with no intervention.

Consent for publication: Not applicable

Funding: This paper was not supported by any funding

Authors' contributions: N.B. collected the data and wrote the manuscript. D.B, G.K, N.S, S.G, V.T, P.Y. and U.B. critically analyzed the data and helped in finalizing the manuscript. All authors reviewed the manuscript

Acknowledgment: We would like to acknowledge the inputs of Ms. Vandana and Ms. Kavita from Microbiology Laboratory and also Dr. Kalpesh Sukhwani for providing critical inputs during preparation of this manuscript.

Conflicts of Interest: Authors do not have any association which may have any conflicts of interest with this manuscript.

Data Availability statement: The data that support the findings of this study are available on request from the corresponding author. The data are not publicly available due to privacy or ethical restrictions 
Novelty \& Impact Statement: Use of multiplex PCRs for diagnosing of respiratory viral infections have been criticized for lack of therapeutic efficacy (therapy change after testing) and poor cost-effectiveness. We demonstrated that diagnosis of respiratory viral infections in neutropenic oncology patients led to antibiotic de-escalation, decrease length of stay, improve infection control practices and saved cost on antibiotics.

\section{References}

1. Litwin, C.M., Bosley, J.G. Seasonality and prevalence of respiratory pathogens detected by multiplex PCR at a tertiary care medical center. Arch Viro/159, 65-72 (2014). https://doi.org/10.1007/s00705013-1794-4

2. Chartrand C, Tremblay N, Renaud C, et al. Diagnostic Accuracy of Rapid Antigen Detection Tests for Respiratory Syncytial Virus Infection: Systematic Review and Meta-analysis. J Clin Microbiol. 2015 Dec;53(12):3738-49. doi: 10.1128/JCM.01816-15. Epub 2015 Sep 9. PMID: 26354816; PMCID: PMC4652120.

3. Freifeld AG, Bow EJ, Sepkowitz KA,et. al; Infectious Diseases Society of America. Clinical practice guideline for the use of antimicrobial agents in neutropenic patients with cancer: 2010 update by the infectious diseases society of america. Clin Infect Dis. 2011 Feb 15;52(4):e56-93. doi: 10.1093/cid/cir073. PMID: 21258094.

4. Snyder M, Pasikhova Y, Baluch A. Early Antimicrobial De-escalation and Stewardship in Adult Hematopoietic Stem Cell Transplantation Recipients: Retrospective Review. Open Forum Infect Dis. 2017;4(4):ofx226. Published 2017 Dec 11. doi:10.1093/ofid/ofx226

5. Petteys MM, Kachur E, Pillinger KE,et. al. Antimicrobial de-escalation in adult hematopoietic cell transplantation recipients with febrile neutropenia of unknown origin. J Oncol Pharm Pract. 2020 Apr;26(3):632-640. doi: 10.1177/1078155219865303. Epub 2019 Aug 18. PMID: 31423947.

6. $\mathrm{CDC}$ Isolation precautions guidelines. Available at https://www.cdc.gov/infectioncontrol/guidelines/isolation/index.html Accessed on 22nd December 2021.

7. Hijano DR, Maron G, Hayden RT. Respiratory Viral Infections in Patients With Cancer or Undergoing Hematopoietic Cell Transplant. Front Microbiol. 2018 Dec 12;9:3097. doi:

10.3389/fmicb.2018.03097. PMID: 30619176; PMCID: PMC6299032.]

8. Torres JP, Labraña Y, Ibañez C,et al. Frequency and clinical outcome of respiratory viral infections and mixed viral-bacterial infections in children with cancer, fever and neutropenia. Pediatr Infect Dis J. 2012 Sep;31(9):889-93. doi: 10.1097/INF.0b013e31825c4b7e. PMID: 22565292.

9. Shinn K, Wetzel M, DeGroote NP,et al. Impact of respiratory viral panel testing on length of stay in pediatric cancer patients admitted with fever and neutropenia. Pediatr Blood Cancer. 2020 Nov;67(11):e28570. doi: 10.1002/pbc.28570. Epub 2020 Sep 2. PMID: 32881268; PMCID: PMC7721999. 
10. Hanson KE, Azar MM, Banerjee R, et al. Molecular Testing for Acute Respiratory Tract Infections: Clinical and Diagnostic Recommendations From the IDSA's Diagnostics Committee. Clin Infect Dis. 2020;71(10):2744-2751. doi:10.1093/cid/ciaa508

11. Beaird OE, Freifeld A, Ison MG et al. Current practices for treatment of respiratory syncytial virus and other non-influenza respiratory viruses in high-risk patient populations: a survey of institutions in the Midwestern Respiratory Virus Collaborative. Transpl Infect Dis. 2016 Apr;18(2):210-5. doi: 10.1111/tid.12510. Epub 2016 Mar 29. PMID: 26923867; PMCID: PMC7169710.

12. Khawaja F, Chemaly RF. Respiratory syncytial virus in hematopoietic cell transplant recipients and patients with hematologic malignancies. Haematologica. 2019;104(7):1322-1331. doi:10.3324/haematol.2018.215152

13. Allen EK, Koeppel AF, Hendley JO, Turner SD, Winther B, Sale MM. Characterization of the nasopharyngeal microbiota in health and during rhinovirus challenge. Microbiome. 2014;2:22. Published 2014 Jun 25. doi:10.1186/2049-2618-2-22

\section{Tables}

\section{Table 1: Various parameters of study population}




\begin{tabular}{|c|c|}
\hline \multicolumn{2}{|l|}{ Variables $(n=57)$} \\
\hline Median Age in years (range) & $40(3-68)$ \\
\hline Males (n/\%) & $38(66.6 \%)$ \\
\hline \multicolumn{2}{|l|}{ Distribution of malignancies } \\
\hline Hematological (leukemia, lymphoma, myeloma) (n/\%) & $45(78.9 \%)$ \\
\hline Solid Tumors (n/\%) & $10(17.5 \%)$ \\
\hline Non-oncological conditions (Thalessemia, aplastic anemia) (n/\%) & $2(3.5 \%)$ \\
\hline Post-stem cell transplant (n/\%) & $14(24.5 \%)$ \\
\hline Median no. of days post transplant (n/\%) & $37(5-400)$ \\
\hline $\begin{array}{l}\text { No. of patients who had severe neutropenia (ANC }<500 \mathrm{~mm}^{3} \text { at the time of } \\
\text { diagnosis of viral infection) }(\mathrm{n} / \%)\end{array}$ & $32(56.1 \%)$ \\
\hline Median no. of days of neutropenia (range) & $7(5-20)$ \\
\hline No. of patients who were managed in ICU (n/\%) & $6(10.5 \%)$ \\
\hline No. of patients who required supplemental oxygen (n/\%) & $15(26.3 \%)$ \\
\hline \multicolumn{2}{|l|}{ Distribution of chest radiological abnormalities } \\
\hline Normal (n/\%) & $27(47.3 \%)$ \\
\hline Ground glass opacities (n/\%) & $22(38.5 \%)$ \\
\hline Nodules (n/\%) & $2(3.5 \%)$ \\
\hline Lobar Consolidation (n/\%) & $4(7.0 \%)$ \\
\hline \multicolumn{2}{|l|}{ Viral pathogen detected } \\
\hline Respiratory Syncytial Virus (RSV) (n/\%) & $21(36.8 \%)$ \\
\hline Influenza (A and B) (n/\%) & $11(19.3 \%)$ \\
\hline Para-influenza virus (n/\%) & $6(10.5 \%)$ \\
\hline Corona Virus (non-SARS COV-2) (n/\%) & $5(8.7 \%)$ \\
\hline Rhinovirus (n/\%) & $11(19.3 \%)$ \\
\hline Human Metapneumovirus & $3(5.2 \%)$ \\
\hline \multicolumn{2}{|c|}{ Therapeutic intervention done after diagnosis of viral pathogen was made } \\
\hline No specific anti-viral added (n/\%) & $36(63.1 \%)$ \\
\hline Oseltamivir added (n/\%) & $11(19.3 \%)$ \\
\hline Oral Ribavirin was added (n/\%) & $5(8.7 \%)$ \\
\hline IVIG plus oral Ribavirin (n/\%) & $1(1.7 \%)$ \\
\hline Only IVIG (n/\%) & $3(5.2 \%)$ \\
\hline Systemic Steroids (n/\%) & $1(1.7 \%)$ \\
\hline \multicolumn{2}{|c|}{ Antibiotic prescription change after diagnosis of viral pathogen } \\
\hline De-escalation of antibiotics (n/\%) & $33(57.8 \%)$ \\
\hline Antibiotics were not escalated (n/\%) & $14(24.5 \%)$ \\
\hline Antibiotics were escalated (n/\%) & $10(17.5 \%)$ \\
\hline Median duration of antibiotics given to the cohort in days (range) & $2(1-14)$ \\
\hline No. of patients who got antibiotics for $>7$ days $(\mathrm{n} / \%)$ & $7(12.2 \%)$ \\
\hline No. of patients in whom co-infection with other identified simultaneously (n/\%) & $13(22.8 \%)$ \\
\hline $\begin{array}{l}\text { No. of patients who were shifted to transmission based isolation precautions } \\
\text { (n/\%) }\end{array}$ & $43(75.4 \%)$ \\
\hline \multicolumn{2}{|l|}{ Outcomes } \\
\hline Median cost of antibiotics saved in INR per patient (range) & $\begin{array}{l}9000(0- \\
67500)\end{array}$ \\
\hline Median Length of stay in days (n/\%) & $6(1-22)$ \\
\hline Mortality (n/\%) & $4(7.0 \%)$ \\
\hline
\end{tabular}


Table 2: Comparison of various parameters between two groups

\begin{tabular}{|c|c|c|c|}
\hline Variables & $\begin{array}{l}\text { Antibiotics } \\
\text { de-escalated } \\
(\mathrm{n}=33\end{array}$ & $\begin{array}{l}\text { Antibiotics } \\
\text { escalated or not de- } \\
\text { escalated }(n=24)\end{array}$ & $\begin{array}{l}\text { p- } \\
\text { value }\end{array}$ \\
\hline Median Age in years & 38 & 39 & 0.90 \\
\hline Males (n/\%) & $23(69.7 \%)$ & $15(62.5 \%)$ & 0.655 \\
\hline \multicolumn{4}{|l|}{ Distribution of malignancies } \\
\hline Hematological Malignancies (n/\%) & $27(81.8 \%)$ & $18(75.0 \%)$ & 0.56 \\
\hline Solid Malignancies (n/\%) & $4(12.1 \%)$ & $5(20.8 \%)$ & 0.37 \\
\hline $\begin{array}{l}\text { No. of patients who had severe neutropenia } \\
\text { (ANC }<500 \mathrm{~mm}^{3} \text { at the time of diagnosis of } \\
\text { viral infection) (n/\%) }\end{array}$ & $14(42.2 \%)$ & $18(75.0 \%)$ & 0.01 \\
\hline Median days of neutropenia & 6 & 7 & 0.81 \\
\hline $\begin{array}{l}\text { No. of patients who were managed in ICU } \\
(\mathrm{n} / \%)\end{array}$ & $0(0.0 \%)$ & $6(25.0 \%)$ & 0.002 \\
\hline \multicolumn{4}{|c|}{ Distribution of chest radiological abnormalities } \\
\hline $\begin{array}{l}\text { Consistent with viral infection (normal, GGOs) } \\
\text { (n/\%) }\end{array}$ & $31(93.9 \%)$ & $19(79.1 \%)$ & 0.09 \\
\hline $\begin{array}{l}\text { Not consistent with viral infection } \\
\text { (consolidation and nodules) (n/\%) }\end{array}$ & $2(6.0 \%)$ & $5(20.8 \%)$ & 0.09 \\
\hline \multicolumn{4}{|l|}{ Viral pathogen detected } \\
\hline RSV (n/\%) & $16(48.4 \%)$ & $5(20.8 \%)$ & 0.03 \\
\hline Rhinovirus (n/\%) & $1(3.0 \%)$ & $10(41.6 \%)$ & 0.0002 \\
\hline Others (Influenza, PIV, Corona, HMPV) (n/\%) & $16(48.4 \%)$ & $9(37.8 \%)$ & 0.40 \\
\hline Co-infection by other pathogen (n/\%) & $0(0.0 \%)$ & $13(54.1 \%)$ & 0.0002 \\
\hline \multicolumn{4}{|l|}{ Outcomes } \\
\hline $\begin{array}{l}\text { Median cost of antibiotics saved in INR per } \\
\text { patient }\end{array}$ & 9620 & 0 & 0.0003 \\
\hline Median Length of stay in days & 3 & 10 & 0.005 \\
\hline Mortality (n/\%) & $0(0.0 \%)$ & $4(16.6 \%)$ & 0.01 \\
\hline
\end{tabular}

\section{Figures}




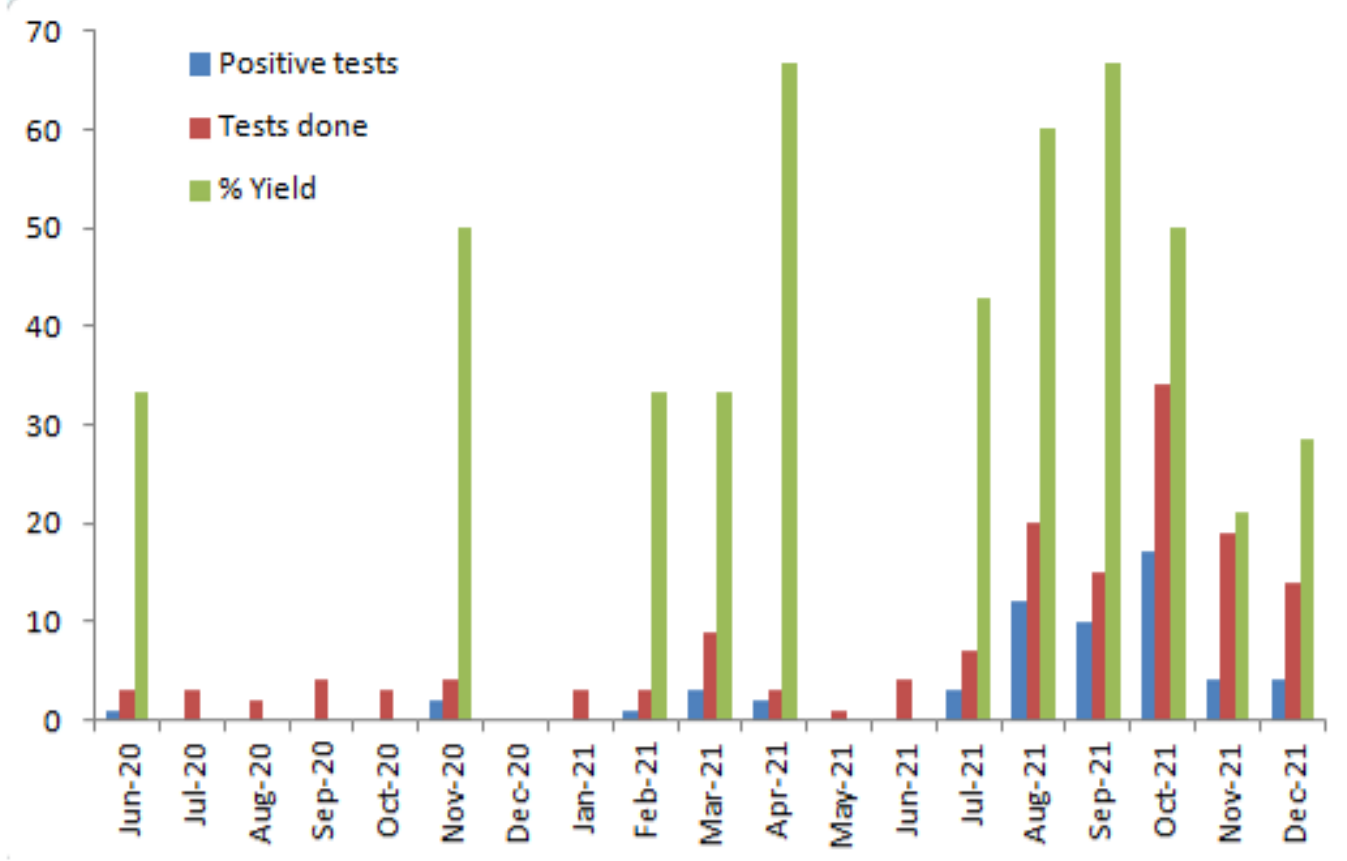

Figure 1

Distribution of positive tests, total number of tests done and positive yield percentage in months across the study period

\section{Supplementary Files}

This is a list of supplementary files associated with this preprint. Click to download.

- SupplementaryTable.docx 